\title{
Left main coronary artery compression due to pulmonary artery aneurysm: Three-year clinical and angiographic follow-up after stenting
}

\author{
Roberto Ramos Barbosa, Afonso Dalmazio Souza Mario, Darlan Dadalt, Nicholas Loureiro de Sá, Renato Giestas \\ Serpa, Tiago de Melo Jacques, Osmar de Araujo Calil, Luiz Fernando Machado Barbosa \\ Santa Casa de Misericórdia de Vitória, Brazil
}

Received: June 10, 2016

DOI: $10.5430 /$ crim.v4n1p64
Accepted: July 28, 2016

Online Published: January 20, 2017

\begin{abstract}
We report a case of a 53-year-old male patient with typical angina and dyspnea with moderate exertion due to extrinsic compression of the left main coronary artery (LMCA) by a pulmonary artery aneurysm. Coronary angiography demonstrated $90 \%$ stenosis of the LMCA ostium. Percutaneous coronary intervention was successfully performed with implantation of a $6.0 \mathrm{~mm} \times 12 \mathrm{~mm}$ bare-metal stent guided by intravascular ultrasound (IVUS). He remained asymptomatic at three-years evaluation, and angiographic and IVUS follow-up showed no significant in-stent neointimal hyperplasia or luminal loss, with excellent apposition and covering of stent struts and minimal luminal area $23.3 \mathrm{~mm}^{2}$ as measured by IVUS.
\end{abstract}

Key Words: Coronary artery disease, Percutaneous coronary intervention, Left main coronary artery, Pulmonary artery hypertension

\section{BACKGROUND}

Pulmonary artery hypertension (PAH) is a progressive vascular disease defined by elevation of pulmonary artery pressure above $25 \mathrm{mmHg},{ }^{[1]}$ and pulmonary vascular resistance may also increase and even reach systemic levels. PAH is classified as primary or secondary to a pulmonary or cardiac underlying disease. ${ }^{[2]}$ A present and more embracing classification includes multiple clinical conditions categorized in five groups, where idiopathic PAH belongs to group $1 .{ }^{[1]}$

Pulmonary vasculature dilatation eventually results in pulmonary artery aneurysm, which may lead to extrinsic compression of the left main coronary artery (LMCA), thus, a rare and potentially reversible cause of chest pain and heart failure, or even sudden death. ${ }^{[3]}$

In this article, we report the clinical and angiographic late follow-up of a patient with the diagnosis of pulmonary artery aneurysm causing compression of the LMCA, submitted to interventional therapy.

\section{CASE REPORT}

A 53-year-old male, with previously known systemic hypertension, presenting chest pain caused by exertion since 2011 with progressive evolution, associated with breathlessness.

Transthoracic echocardiogram (TTE) revealed pulmonary artery aneurysm (trunk $67 \mathrm{~mm}$, right branch $60 \mathrm{~mm}$ and left branch $40 \mathrm{~mm}$ ), moderate pulmonary valve regurgitation,

\footnotetext{
${ }^{*}$ Correspondence: Roberto Ramos Barbosa; Email: roberto.rb@cardiol.br; Address: Jairo de Matos Pereira St., 780/1001, Praia da Costa, Vila Velha,
} ES, Brazil. 
moderate augmentation of right chambers and PAH classified as mild to moderate (systolic pulmonary artery pressure $43 \mathrm{mmHg}$ ).

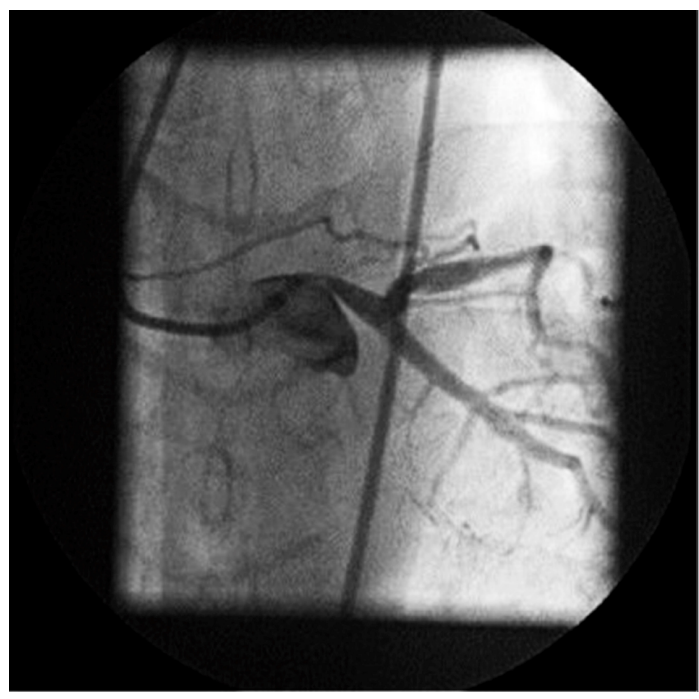

Figure 1. Coronary angiography: severe stenosis in left main coronary artery ostium due to extrinsic compression from an aneurysm of the pulmonary artery

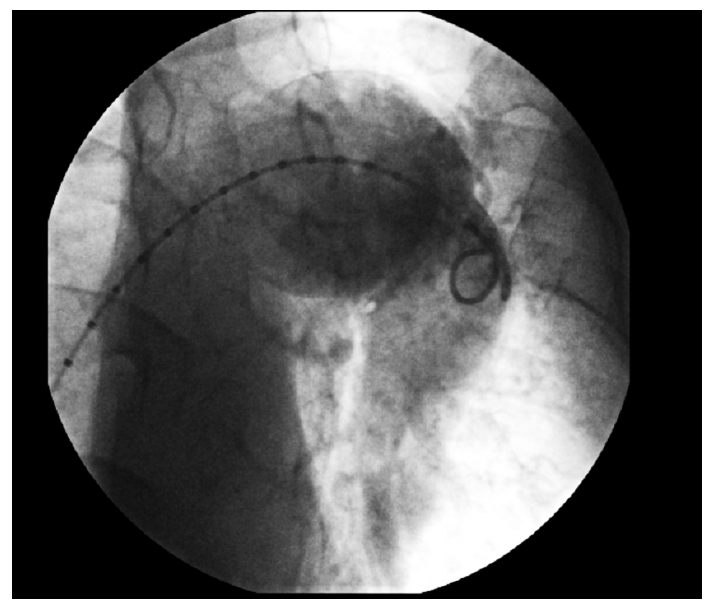

Figure 2. Pulmonary angiography during right cardiac catheterization: severe dilatation of the pulmonary artery and its main branches, associated to pulmonary artery hypertension

In April 2011, the patient underwent coronary angiography, which demonstrated a $90 \%$ stenosis of the LMCA ostium, with a suggesting image of extrinsic compression by the trunk of the pulmonary artery (coronary "folding" with no aspect of atherosclerotic disease) (see Figure 1), then confirmed by computed tomography angiography (aneurysmatic dilatation of pulmonary artery-trunk $70 \mathrm{~mm}$, right branch $64 \mathrm{~mm}$ and left branch $46 \mathrm{~mm}$ ). The right coronary artery and the branches of the left coronary artery were free from obstrucPublished by Sciedu Press tive disease. Right cardiac catheterization was performed and revealed an elevated systolic pulmonary artery pressure $(51 \mathrm{mmHg})$ and severe dilatation of the pulmonary artery and its branches (see Figure 2), with mild right ventricle systolic disfunction.

The option of surgical myocardial revascularization was considered and then eliminated, since perioperative risk was considered uncertain, as well as the strategy of surgically approaching the pulmonary artery aneurysm.

Facing the feature of limiting chest pain and the presence of a critical stenosis in the LMCA involving its ostium and body, we decided to perform a percutaneous coronary intervention (PCI) with stenting.

\section{Procedure}

PCI was performed in May of 2011, through a femoral access, with a JL 7F guiding-catheter (Terumo, Tokyo, Japan) and a 0.014" PT2 guidewire (Boston Scientific, MA, United States). Pre-dilatation with compliant $3.0 \mathrm{~mm} \times 10 \mathrm{~mm}$ Pantera balloon (Biotronik, Lake Oswego, United States) was followed by implantation of a $6.0 \mathrm{~mm} \times 12 \mathrm{~mm}$ Express SD bare-metal stent (Boston Scientific, MA, United States) at 14 ATM (see Figure 3A). Post-dilatation with the stent $6.0 \mathrm{~mm} \times 12 \mathrm{~mm}$ balloon was performed and guided by intravascular ultrasound (IVUS), with expansion until 20 ATM. Coronary angiography and immediate control IVUS showed procedure success and excelent angiographic result, with great expansion and stent struts apposition, limited to the LMCA from its ostium (see Figure 3B).

The decision for a bare-metal stent came through the uncertainess concerning the patient's adherence to prolonged antiplatelet therapy, and also the possible future indication for oral anticoagulation after ethiology definition of the $\mathrm{PAH}$, which might increase bleeding risk when associated with dual antiplatelet therapy in the first year.

\section{FOLLOW-UP}

New TTE in 2012 did not reveal significant modifications concerning the PAH or the measures of the pulmonary artery aneurysm. The patient presented relief of chest pain and breathlessness, and returned to daily activities. Sildenafil citrate was initiated for treatment of the PAH and remains until present, with good response.

Asymptomatic, the patient was submitted to protocol coronary angiography with IVUS in December 2014, which revealed pervious stent in LMCA, without significant neointimal hyperplasia or luminal loss, with excelent struts apposition and covering at IVUS imaging (see Figure 4). The in-stent minimal luminal area at IVUS was $23.4 \mathrm{~mm}^{2}$. 

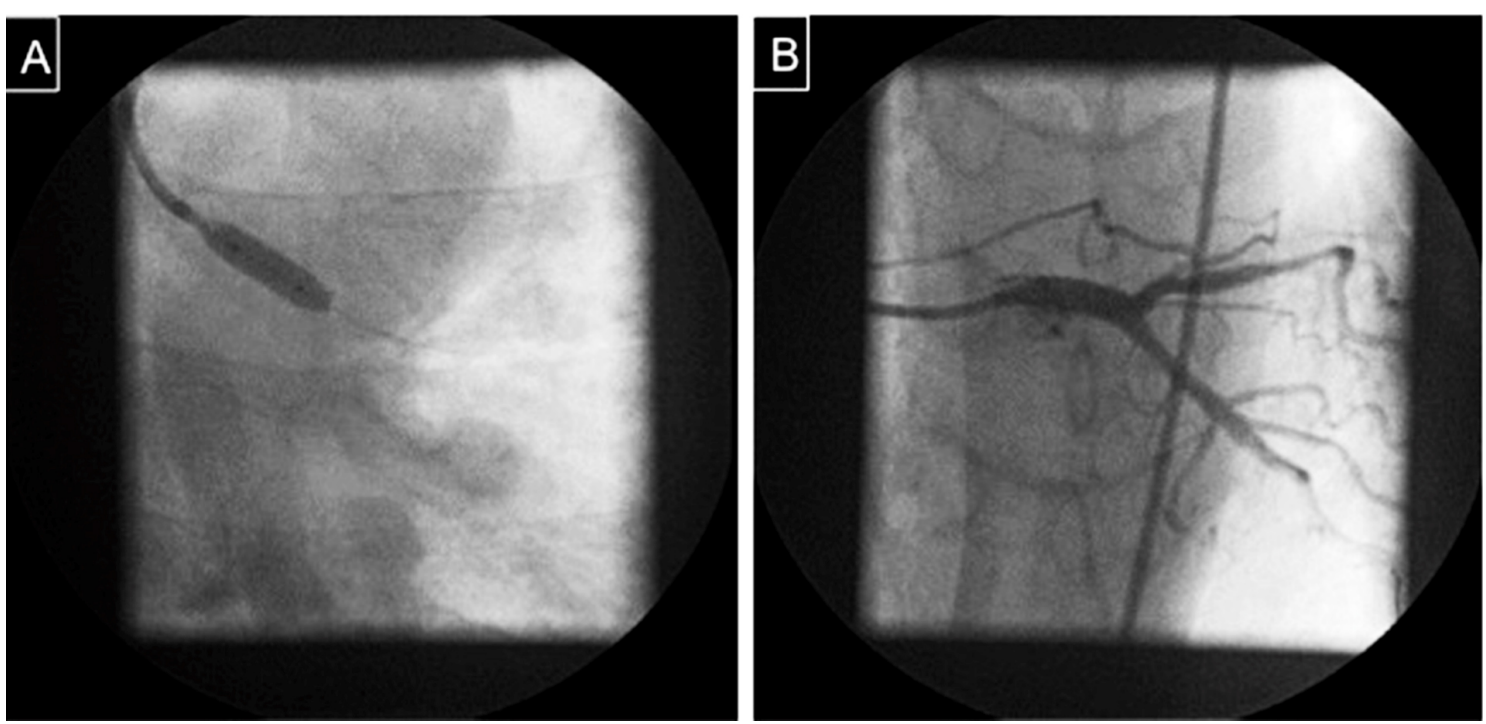

Figure 3. A. Percutaneous coronary intervention (PCI) with implantation of a $6.0 \mathrm{~mm} \times 12 \mathrm{~mm}$ stent in the left main coronary artery; B. Immediate post-PCI angiographic result

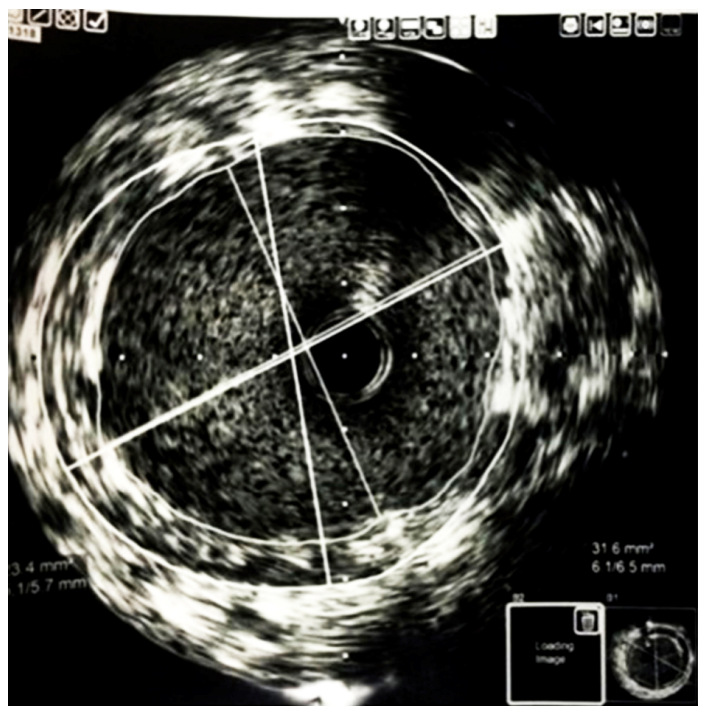

Figure 4. Intravascular ultrassound obtained three years after stent implantation in the left main coronary artery

\section{Discussion}

The extrinsic compression of the LMCA from distension of the trunk of the pulmonary artery was first described by Corday et al. in 1957. ${ }^{[4]}$

Despite the advances observed in percutaneous and surgical techniques for myocardial revascularization, the best management of patients with chest pain caused by extrinsic compression of the LMCA is yet to be defined.

Coronary artery bypass graft (CABG) surgery and PCI are, at present, feasible and comparable, concerning obstructive disease in the LMCA. Due to the fact that the pulmonary artery aneurysm usually compress the ostium and proximal segment of the LMCA, and because of the slightly elevated perioperative risk of patients with $\mathrm{PAH},{ }^{[5]} \mathrm{PCI}$ with stent implantation in the LMCA is often considered the strategy of choice. ${ }^{[3,6]}$

The type of coronary stent to be implanted is another matter of controversy, once the PAH evolution might be unpredictable and its etiology may vary. These factors, especially when unclear, are potential confounders and may interfere in dual antiplatelet therapy, if vascular thrombosis within the pulmonary territory occurred or is at risk. Moreover, whether the cause of PAH has not been defined, oral anticoagulation may be considered when chronic pulmonary embolism is suspected. ${ }^{[1]}$ In both situations, for decisions such as duration of dual antiplatelet therapy and its association or not to oral anticoagulants, individual management is mandatory. These features reflect on the type of coronary stent chosen - whether it is a drug-eluted stent or not - depending on the risk of bleeding and thrombotic events.

The first description of percutaneous approach within this context was made in 2001 by Rich et al., who reported successful LMCA stenting-PCI in two patients with primary $\mathrm{PAH}$ and associated extrinsic LMCA compression. ${ }^{[7]}$ Since then, other authors reported angiographic and clinical followup success, confirming favorable outcomes in such cases with the technique. ${ }^{[8-14]}$

In all reported cases, as well as in the present report, the stenosis in LMCA was not followed by obstructive disease in other coronary territories. The absence of atherosclerotic coronary disease is a sign for suspicion of extrinsic LMCA compression, although aortic diseases, such as systemic vas- 
culitis, syphilitic aortitis or rheumatologic diseases, shall be excluded. ${ }^{[3]}$

Long-term follow-up of patients undergoing LMCA PCI are rare in medical literature, when the cause of stenosis is extrinsic compression from a pulmonary artery aneurysm. Noteworthy, the reported case presents a three-year followup, with coronary angiography and IVUS, after the index procedure. Most reports of similar cases present up to 12month clinical follow-up. ${ }^{[5,6,14]}$

In today's clinical and interventional practice, LMCA PCI is feasible and has been proven safe, especially in situations of elevated clinical risk and/or favorable angiographic characteristics and low anatomic complexity. ${ }^{[15]}$ The presence of PAH and aneurysm of the pulmonary artery as the relying cause of the coronary obstruction establish a rare and difficult case, demanding critical therapeutic decisions and individualized clinical follow-up. IVUS, as shown in recent literature, can play an importante role on PCI guiding, leading to successful catheter-based therapy of LMCA extrinsic compression. ${ }^{[16]}$ IVUS is a largely studied and available tool that represents a solid diagnosing resource, also valuable for treatment orientation and follow-up in such cases.

Other coronary imaging modalities, such as optical coherence tomography, may be useful and have been described in the evaluation after LMCA stenting motivated by extrinsic compression from a pulmonary artery aneurysm..$^{[14]}$

\section{CONFlicts OF InTEREST Disclosure}

The authors declare no conflicts of interests related to this manuscript.

\section{REFERENCES}

[1] 2015 ESC/ERS Guidelines for the diagnosis and treatment of pulmonary hypertension. The Joint Task Force for the Diagnosis and Treatment of Pulmonary Hypertension of the European Society of Cardiology (ESC) and the European Respiratory Society (ERS). Endorsed by: Association for European Paediatric and Congenital Cardiology (AEPC), International Society for Heart and Lung Transplantation (ISHLT). Eur Heart J. 2015; 317: 1-58. PMid:15302363 https://doi.org/10.1016/j.ccl.2004.04.004

[2] Channick R, Williamson TL. Diagnosis and treatment of pulmonary arterial hypertension. Cardiol Clin. 2004; 22(3): 441-52.

[3] Lee MS, Oyama J, Bhatia R, et al. Left main coronary artery compression from pulmonary artery enlargement due to pulmonary hypertension: A contemporary review and argument for percutaneous revascularization. Catheter Cardiovasc Interv. 2010; 76(4): 543-50. PMid:20506194 https://doi.org/10.1002/ccd. 22592

[4] Corday E, Gold H, Kaplan L. Coronary artery compression: an explanation for the cause of coronary insufficiency in pulmonary hypertension. Trans Am Coll Cardiol. 1957; 7: 93-103. PMid:13409433

[5] Jurado-Román A, Hernández-Hernández F, Ruíz-Cano MJ, et al. Compression of the left main coronary artery by a giant pulmonary artery aneurysm. Circulation. 2013; 127: 1340-134. PMid:23529535 https://doi.org/10.1161/CIRCULATIONAHA.112.152199

[6] Caldera AE, Cruz-Gonzalez I, Bezerra HG, et al. Endovascular therapy for left main compression syndrome: Case report and literature review. Chest. 2009; 135(6): 1648-50. PMid:19497900 https://doi.org/10.1378/chest.08-2922

[7] Rich S, McLaughlin VV, O'Neill W. Stenting to reverse left ventricular ischemia due to left main coronary artery compression in primary pulmonary hypertension. Chest. 2001; 120: 1412-1415. PMid:11591592 https://doi.org/10.1378/chest.120.4.14 12

[8] Lindsey JB, Brilakis ES, Banerjee S. Acute coronary syndrome due to extrinsic compression of the left main coronary artery in a patient with severe pulmonary hypertension: successful treatment with percutaneous coronary intervention. Cardiovasc Revasc Med. 2008; 9: 47-51. PMid:18206638 https://doi.org/10.1016/j.carr ev.2007.07.003
[9] Dodd JD, Maree A, Palacios I, et al. Images in cardiovascular medicine: left main coronary artery compression syndrome; evaluation with 64-slice cardiac multidetector computed tomography. Circulation. 2007; 115: e7-e8. PMid:17200448 https ://doi.org/ 10.1161/CIRCULATIONAHA. 106.645622

[10] Varela SG, Orbe PMM, Villa JA, et al. Stenting in primary pulmonary hypertension with compression of the left main coronary artery. Rev Esp Cardiol. 2004; 57: 695-698.

[11] Dubois CL, Dymarkowski S, Cleemput JV. Compression of the left main pulmonary artery in a patient with the Eisenmenger syndrome. Eur Heart J. 2007; 28: 1945. PMid:17311829 https : //doi .org/ 10.1093/eurheartj/eh1556

[12] Vaseghi M, Lee JS, Currier JW. Acute myocardial infarction secondary to left main coronary artery compression by pulmonary artery aneurysm in pulmonary arterial hypertension. J Invasive Cardiol. 2007; 19: 375-377.

[13] Godfrey A, Cajigas HR. A 55-year-old woman with pulmonary hypertension, worsening dyspnea, and chest pain. Chest. 2014; 145(3): 642-5. https://doi.org/10.1378/chest.13-1735

[14] Ogiso M, Serizawa N, Kamishima K, et al. Percutaneous coronary intervention for left main compression syndrome due to severe idiopathic pulmonary arterial hypertension: one year follow-up using intravascular imaging. Internal Medicine. 2015; 54: 801-804. PMid:25832945 https://doi.org/10.2169/internalmedici ne. 54.3528

[15] 2011 ACCF/AHA/SCAI Guideline for percutaneous coronary intervention: Executive summary: A report of the American College of Cardiology Foundation/American Heart Association Task Force on Practice Guidelines and the Society for Cardiovascular Angiography and Interventions. Circulation. 2011; 124: 2574-2609. PMid:22064598 https://doi.org/10.1161/CIR.0b013e3182 $3 a 5596$

[16] Pan HC, Wang KY, Liang KW. Left main coronary artery stenting to relieve extrinsic compression by a giant pulmonary artery aneurysm in a patient with idiopathic pulmonary artery hypertension. Heart Lung Circ. 2016: S1443-9506(16)30040-3. 